\title{
Maternal rectal temperature and fetal heart rate responses to upright cycling in late pregnancy
}

\author{
M E O’Neill
}

\begin{abstract}
Objective-To assess maternal rectal temperature and fetal heart rate responses to dynamic exercise.

Methods-11 healthy women with low risk pregnancies completed three separate upright cycling tests at 34 to 37 weeks gestation: $15 \mathrm{~min}$ at $62.5 \mathrm{~W}$ (mean maternal heart rate [MHR] 138 beats $\cdot \mathrm{min}^{-1}$ (test $\mathrm{A}$ ); $15 \mathrm{~min}$ at $87.5 \mathrm{~W}$ (MHR 156 beats $\cdot \mathrm{min}^{-1}$ ) (test B); and 30 min at $62.5 \mathrm{~W}$ (MHR 142 beats $\cdot \mathrm{min}^{-1}$ ) (test $C$ ). Rectal temperature and fetal heart rate were measured.

Results-Mean temperature increase after tests $B$ and $\mathrm{C}$ [by $0.4(\mathrm{SD} 0 \cdot 1)^{\circ} \mathrm{C}$ ] was greater than after test $A\left[0 \cdot 2(0 \cdot 1)^{\circ} \mathrm{C}\right]$ $(P<0 \cdot 001)$. Fetal heart rate, measured in the recovery period immediately after exercise, increased significantly only after tests $B$ and $C(P<0 \cdot 01)$. Exercise related changes in temperature and fetal heart rate weakly correlated in tests $B(P<0.02)$ and $C(P<0.01)$.
\end{abstract}

Conclusions-Temperature and fetal heart rate changes were more marked after higher intensity (test B) or longer duration exercise (test $C$ ) compared with moderate exercise, but none of the tests caused adverse fetal heart rate changes (decrease in accelerations, bradycardia, or decelerations) or individual temperatures above $38^{\circ} \mathrm{C}$.

(Br F Sports Med 1996;30:32-35)

Key terms: pregnancy; exercise; exertion; fetal heart rate; temperature

There are several potential concerns about exercise in pregnancy such as an increase in fetal temperature, fetal hypoglycaemia, and a decrease in uterine artery blood flow and oxygen delivery to the fetus. ${ }^{1}$ Physical activity can increase maternal core temperature, which in turn can cause the fetal temperature to rise. Repeated episodes of marked hyperthermia in animals have been associated with adverse effects such as an increased incidence of fetal malformations (when exposed early in pregnancy), ${ }^{1}$ decreased postnatal learning ability, ${ }^{2}$ and decreased birthweight (when exposure occurs in the last half of pregnancy). ${ }^{3}$ To try to prevent adverse fetal effects, it has been recommended that the core temperature in pregnant women should not exceed $38^{\circ} \mathrm{C}$ when exercising. ${ }^{4-6}$

When non-pregnant women were instructed to get out of a hot spa as soon as they felt too warm, none left before their core temperature reached $39^{\circ} \mathrm{C}$ or more. ${ }^{7}$ If pregnant women behave similarly, they might not feel uncomfortable when their temperature reaches $38^{\circ} \mathrm{C}$ and would be unlikely to stop exercising spontaneously at this temperature. It is therefore important to evaluate if the maternal core temperature responses to some specific exercise prescriptions are likely to be above the recommended levels.

Because the fetal effects of exercise and other physiological changes cannot be examined directly, the fetal heart rate is often used as an indirect measure of change in the fetal environment. There is controversy about whether fetal heart rate response to physical activity is influenced by exercise intensity ${ }^{8}$ or not, ${ }^{9-10}$ even though it is duration dependent. $^{8} 11$

In this study I examined whether more marked changes in temperature or fetal heart rate will occur as the duration or intensity of exercise increases. The maternal core temperature and fetal heart rate responses to three combinations of exercise intensity and duration were studied. The 1994 guidelines of the Royal Australian College of Obstetricians and Gynaecologists ${ }^{5}$ and the Australian Sports Medicine Federation ${ }^{6}$ recommend that pregnant women should not exercise for more than 15 to 20 minutes at a maternal heart rate of 140 beats $\mathrm{min}^{-1}$. This is similar to the 1985 guidelines of the American College of Obstetricians and Gynecologists ${ }^{4}$ and corresponds to the lowest combination of intensity and duration examined in this study. The other two tests are also of particular relevance because the guidelines of the American College of Obstetricians and Gynecologists became more liberal in $1994^{12}$ and so more pregnant women may want to exercise at a higher intensity or for a more prolonged period.

\section{Methods}

SUBJECTS

Healthy non-smokers with low risk singleton pregnancies were tested at 34 to 37 weeks gestation. They did not have any absolute or relative contraindications to exercise. ${ }^{4}$ They did not require medication apart from haematinics and vitamin supplements. All gave informed consent. They were all Caucasian and resident in Auckland, New Zealand, where the tests were conducted. Twelve women were recruited but only 11 women completed all three tests. The 12 th volunteer was unable to complete test $\mathrm{C}$ due to muscle fatigue and so her results were excluded from the statistical analysis. 
The women $(n=11)$ were tested at $35 \cdot 8(\mathrm{SD}$ $1 \cdot 1)$ weeks of gestation. Their average age was $30.3(3 \cdot 3)$ years. Their mean weight was $68 \cdot 1(4 \cdot 2) \mathrm{kg}$ and their height was $1 \cdot 63(0 \cdot 5) \mathrm{m}$. Eight were primiparous and three multiparous. A wide range of fitness levels was represented. There were four "trained" subjects who had been exercising for the previous 10 weeks or more at least thrice weekly, for 30 minutes or more, at an intensity that was high enough to cause shortness of breath and perspiration. There were five "sedentary" subjects who never participated in an exercise session. The remaining two women sometimes exercised but not enough to be considered "in training".

\section{EXERCISE PROTOCOL}

Participants had a light snack approximately $2 \mathrm{~h}$ before exercising. In the $30 \mathrm{~min}$ rest period, they filled out questionnaires and were familiarised with the protocol, equipment, and laboratory conditions. Dry temperature was maintained at $21.5-23.0^{\circ} \mathrm{C}$ by air conditioning.

In test $A$, subjects exercised on an upright bicycle ergometer for $15 \mathrm{~min}$ at $62.5 \mathrm{~W}$. They then rested for $1 \mathrm{~h}$ before cycling for $15 \mathrm{~min}$ at $87.5 \mathrm{~W}$ (test $\mathrm{B}$ ). The following day, they cycled at $62.5 \mathrm{~W}$ for $15 \mathrm{~min}$, stopped briefly for up to $1.5 \mathrm{~min}$ to allow fetal heart rate measurements to be made to assess day to day variability, and then continued for another $15 \mathrm{~min}$. Hence they cycled for $30 \mathrm{~min}$ in total (test C). All measurements were made in the upright sitting position. Subjects were encouraged to continue pedalling gently in recovery to prevent venous pooling.

Maternal heart rate was recorded at rest and in the last $30 \mathrm{~s}$ of exercise (Hewlett-Packard 1405A electrocardiogram). Maternal rectal temperature was examined immediately before and after cycling with a Becton Dickinson digital thermometer. (According to a personal communication with Becton Dickinson, Knoxfield, Australia, it has an accuracy of $\pm 0.02^{\circ} \mathrm{C}$.) The temperature values were recorded to the nearest $0 \cdot 1^{\circ} \mathrm{C}$. Rectal temperature was used as the measure of maternal core temperature because this study was interested in local heat changes close to the fetus.

Baseline fetal heart rate was measured at rest and at $1,5,10,15$, and $20 \mathrm{~min}$ into the recovery period using a cardiotocogram with autocorrelation (Hewlett-Packard 8040A). The incidence of fetal heart rate accelerations (defined as a transient increase in rate by at least 15 beats. $\mathrm{min}^{-1}$ for $15 \mathrm{~s}$ or more) was assessed in the $15 \mathrm{~min}$ periods before and after exercise.

\section{DELIVERY DATA}

Each baby $(n=11)$ was born between 264 and 288 days of gestation [mean $280(8)$ d], had a birthweight between the 10th and 90th centiles for gestational age [mean birthweight $3 \cdot 4(0 \cdot 5)$ $\mathrm{kg}$, did not require admission to neonatal intensive care, and survived the first 28 days after delivery. There were five males and six females.
STATISTICS

The results of the 12th volunteer were excluded from the statistical analysis because she did not complete all three tests. For all statistical tests, the level of significance was set at $\mathrm{P}<0.05$. Paired $t$ tests were used to compare the following results: (1) baseline maternal measurements and the changes with exercise in tests $\mathrm{A}, \mathrm{B}$, and $\mathrm{C}$ (for fetal heart rate, the rate at $1 \mathrm{~min}$ into recovery was used); (2) if exercise-induced changes were significant; (3) if there were differences in peak maternal heart rate achieved in tests $A$ and $C$, and (4) the change in fetal heart rate after test $A$ versus that seen after cycling for the first $15 \mathrm{~min}$ in test C. Repeated measures analysis with the NewmanKeuls test applied post hoc was used to determine when fetal heart rate was different from pre-exercise levels. The correlation between exercise related changes in maternal core temperature and fetal heart rate was assessed with Pearson's test.

\section{Results}

EXERCISE RESPONSE

The results are shown in the table. There were no differences in the maternal and fetal baseline values for tests $A, B$, and $C$. There was no difference in maternal heart rate achieved in tests $\mathrm{A}$ and $\mathrm{C}$, which indicates that they were of similar intensity.

The maternal rectal temperature increased after exercise in all three tests $(P<0.01)$. Greater increases in temperature were seen after exercising at a higher intensity (test B versus test $A ; P<0.001)$ or for a longer duration (test $C$ versus test $A ; P<0.001$ ). The change in temperature was similar in tests $B$ and $C(P>0.05)$.

The maximum individual increases in temperature were $0.3^{\circ}, 0.5^{\circ}$, and $0.6^{\circ} \mathrm{C}$ in tests $A, B$, and $C$ respectively. None of the individual temperatures exceeded $38^{\circ} \mathrm{C}$. The highest individual temperature observed was $37 \cdot 7^{\circ} \mathrm{C}$ after test $\mathrm{C}$.

The increase in mean fetal heart rate was statistically significant after moderate duration, high intensity exercise (test $B, P<0.01$ ) and 30 min of moderate intensity cycling (test $C$, $P<0.01$ ) but not after moderate duration, moderate intensity cycling (test A).

Compared with test $\mathrm{A}$, the increases in fetal heart rate observed at $1 \mathrm{~min}$ after exercise were greater in tests $B$ and $C(P<0.05)$. They were short lived, resolving by $20 \mathrm{~min}$ into recovery. There were no episodes of fetal bradycardia or fetal heart rate decelerations after exercise. The frequency of fetal heart rate accelerations was similar before and after physical activity.

In test $A$, the change in fetal heart rate at 1 min postexercise did not correlate significantly with the change in temperature $\left(r^{2}=15 \%\right)$. In contrast, it correlated with the change in temperature after test $\mathrm{B}\left(r^{2}=58 \%\right.$, $\mathrm{P}<0.02)$ and test $\mathrm{C}\left(r^{2}=68 \%, \mathrm{P}<0.01\right)$.

The difference between the change in fetal heart rate after exercising for $15 \mathrm{~min}$ on two different days (test $\mathrm{A}$ versus results obtained after $15 \mathrm{~min}$ of exercise in test C) was $1 \cdot 8(8 \cdot 2)$ 
Influence of intensity and duration on maternal and fetal responses to cycling. Values are means (SD)

\begin{tabular}{|c|c|c|c|c|}
\hline & & Test $A$ & Test $B$ & Test $C$ \\
\hline MHR & $\begin{array}{l}\text { Rest } \\
\text { Ex }\end{array}$ & $\begin{array}{r}87(14) \\
138(16)\end{array}$ & $\begin{array}{r}87(12) \\
156(14)\end{array}$ & $\begin{array}{r}87(13) \\
142(16)\end{array}$ \\
\hline Temp $\left({ }^{\circ} \mathrm{C}\right)$ & $\begin{array}{l}\text { Rest } \\
\text { Post-ex } \\
\text { change }\end{array}$ & $\begin{array}{r}37 \cdot 2(0 \cdot 1) \\
37 \cdot 3(0 \cdot 2) \\
0.2(0 \cdot 1)\end{array}$ & $\begin{array}{c}37 \cdot 1(0 \cdot 1) \\
37 \cdot 5(0 \cdot 1) \\
0.4(0 \cdot 1)\end{array}$ & $\begin{array}{r}37.1(0 \cdot 1) \\
37.5(0 \cdot 1) \\
0.4(0 \cdot 1)\end{array}$ \\
\hline FHR & $\begin{array}{l}\text { Rest } \\
\text { Post-ex } \\
\text { change } \\
1 \mathrm{~min} \\
5 \mathrm{~min} \\
10 \mathrm{~min} \\
15 \mathrm{~min} \\
20 \mathrm{~min}\end{array}$ & $\begin{array}{l}3.1(6.8) \\
2.7(5.6) \\
1.9(5.4) \\
0.8(4 \cdot 8) \\
1.8(4 \cdot 7)\end{array}$ & $\begin{array}{r}10.6(4.0) \\
13.2(4.8) \\
11.8(5.0) \\
7.8(4 \cdot 8) \\
3.6(4.6)\end{array}$ & $\begin{array}{r}12 \cdot 6(3 \cdot 4) \\
13.0(5 \cdot 2) \\
11.2(4 \cdot 4) \\
7 \cdot 6(5 \cdot 6) \\
4 \cdot 4(6 \cdot 2)\end{array}$ \\
\hline Accel & $\begin{array}{l}\text { Rest } \\
\text { Post-ex } \\
\text { change }\end{array}$ & $\begin{array}{r}0.32(0.30) \\
-0.11(0.40)\end{array}$ & $\begin{array}{r}0.27(0.35) \\
-0.15(0.37)\end{array}$ & $\begin{array}{r}0.35(0.27) \\
-0.16(0.42)\end{array}$ \\
\hline
\end{tabular}

MHR, maternal heart rate in beats $\mathrm{min}^{-1}$; Ex, exercise; Post-ex, postexercise; Temp, maternal rectal temperature; FHR, fetal heart rate in beats $\mathrm{min}^{-1}$; Accel, fetal heart rate accelerations $\cdot \mathrm{min}^{-1}$. in exercisers during pregnancy but this has not yet been established.

Clothing is another important consideration. The subjects in the present study all wore loose clothing. Most wore a tee-shirt (usually made of mixed cotton and polyester) and loose shorts or a skirt. The temperature increase would be expected to be greater if warmer clothing was worn when exercising such as a track suit.

Hence there are many variables that can influence the core and rectal temperature responses to exercising at a specific intensity and duration. If there are concerns that exercise is increasing rectal temperature too much, it can be measured objectively with a thermometer after carefully teaching pregnant women how to use and insert it properly. This method was well tolerated by all participants in this study.

My study lends support for the view that fetal heart rate response to physical activity is beats $\min ^{-1}$, which was not significant $(\mathrm{P}>0.05)$. dependent on intensity ${ }^{8}$ and duration. ${ }^{811}$ However, intensity effects may only be apparent with moderate duration or prolonged exercise, since they have not been seen in response to studies using incremental exercise with short stages of six minutes or less, ${ }^{1011}$ Another study that concluded that intensity did not influence fetal heart rate ${ }^{9}$ failed to take into account the confounding effects of using different subjects for each intensity level tested, and the high intensity test was performed for a shorter duration than the low and medium intensity tests.

It is important to note that the larger increase in fetal heart rate seen after exercising at a higher intensity or longer duration was short lived, lasting for less than 20 minutes. It is unlikely to reflect a physiological change causing fetal distress because there were no accompanying changes such as fetal bradycardia, heart rate decelerations, or a decrease in heart rate accelerations. In my study, fetal heart rate was measured immediately after exercise; it was not measured during exercise because artefacts due to movement with vigorous pedalling can interfere with data collection. ${ }^{17}$ An earlier study overcame this problem by measuring fetal heart rate during and after exercise with echocardiography. ${ }^{18}$ This showed that postexercise fetal heart rate values did reflect exercise values and that adverse findings such as fetal bradycardia were more likely to occur in recovery than during exercise.

In isolated human fetal hearts removed in early pregnancy, fetal heart rate increases by 10 beats $\cdot \min ^{-1}$ for every $1^{\circ} \mathrm{C}$ rise in temperature until fetal bradycardia occurs at $40^{\circ} \mathrm{C} .^{19}$ Furthermore, fetal heart rate correlates with fetal scalp temperature during delivery in humans. ${ }^{20}$ However, Clapp et $a l^{8}$ found that exercise related changes in fetal heart rate did not correlate with accompanying changes in maternal rectal temperature. The present study found a weak association between exercise related changes in fetal heart rate and temperature when mean core temperature was increased by $0.4^{\circ} \mathrm{C}$. These results suggest that a threshold may need to be passed before same workload causes a lower increase in core temperature in athletes than in sedentary controls $^{14}$. Exercise induced increases in temperature might similarly be relatively lower 
temperature increases influence fetal heart rate and that fetal heart rate changes with exercise are not solely due to temperature changes. A previous study found that fetal heart rate can increase after jogging, even when there is no change in temperature. ${ }^{21}$ Other hypotheses about why fetal heart rate changes with exercise also need to be considered. Although an exercise related increase in circulating maternal catecholamines was postulated to be a cause, catecholamine concentrations have not been found to correlate with fetal heart rate changes. ${ }^{813}$ A second postulated mechanism based on ovine studies is that exercise during pregnancy may decrease uteroplacental flow. ${ }^{22}$ In pregnant humans, the estimated uterine artery blood flow during brief exercise periods of four minutes decreased only at maximal physical activity and was not related to fetal heart rate changes. ${ }^{10}$ The influence of different combinations of exercise intensity and duration on the uterine artery blood flow on fetal heart rate still requires further evaluation. The precise cause for the increase in fetal heart rate in response to exercise is not yet clear.

Because there is a paucity of data about the chronic effects of repeated exercise using specific regimes, such as those tests in this study, additional studies would be of value. One study ${ }^{23}$ showed that babies born of women who cycled thrice weekly for 14 to 25 minutes at around 150 beats $\mathrm{min}^{-1}$ had a lower mean birthweight (by about $100 \mathrm{~g}$ ) compared with the sedentary control group, but this difference did not achieve statistical significance.

In conclusion, when prescribing exercise, it is important to specify both the intensity and the duration because it is the combination that determines the temperature response. When considering acute temperature and fetal heart rate effects, no contraindications to cycling for 30 minutes at a maternal heart rate of around 140 beats $\mathrm{min}^{-1}$ or exercising for 15 minutes at a rate of around 155 beats $\cdot \mathrm{min}^{-1}$ were found.

This study was performed when MEO'N was a $\mathrm{PhD}$ student of the University of Sydney working and writing up away from the university in Auckland, New Zealand. Greenlane Hospital and the National Women's Hospital, Auckland, kindly loaned equipment. Dr David Coles and Associate Professor Barry McGrath provided support and constructive feedback.
1 Bell R, O'Neill M. Exercise and pregnancy. Birth 1994;21: 85-95.

2 Jonson KM, Lyle JG, Edwards MJ, Penny RHC. Effect of prenatal heat stress on brain growth and seria discrimination reversal learning in the guinea pig. Brain Res Bull 1976;1:133-50.

3 Bell AW, Wilkening RB, Meschia G. Some aspects of placental function in chronically heat-stressed ewes. $\mathcal{F} \mathrm{Dev}$ Physiol 1987;9:17-29.

4 American College of Obstetricians and Gynecologists. Home exercise program: exercise during pregnancy and the postexercise program: exercise durng pregnancy and the post-
natal period. Washington DC: American College of natal period. Washington DC: America

5 Bell R. Exercise and pregnancy. Royal Australian College of Obstetricians and Gynaecologists continuing education 1993; Resource unit 106:106.1-6.

6 Medicine and Science for Women in Sport committee. Participation of the pregnant athlete in contact and collision sports. Aust Sports Med Fed 1994; May: Guideline 1:1-7.

7 Ridge BR, Budd GM. How long is too long in a spa pool? NEngl f Med 1990;323:835.

8 Clapp JF, Little KD, Capeless EL. Fetal heart rate response to sustained recreational exercise. Am f Obstet Gynecol 1993;168:198-206.

9 Artal R, Rutherford S, Romem Y, Kammula RK, Dorey FJ, Wiswell RA. Fetal heart rate responses to maternal exercise. Am f Obstet Gynecol 1986;155:729-33.

10 Erkkola RU, Pirhonen JP, Kivijarvi AK. Flow velocity waveforms in uterine and umbilical arteries during waveforms in uterine and umbilical arteries during submaximal bicycle ex

11 Sady SP, Carpenter MW. Aerobic exercise during pregnancy: special considerations. Sports Med 1989;7: 357-75.

12 American College of Obstetricians and Gynecologists. Exercise during pregnancy and the postpartum period Am Coll Obstet Gynecol Tech Bull 1994 (Feb);189:1-5.

13 O'Neill ME. Maternal and fetal responses to exercise during the last half of pregnancy and implications for exercise prescription. Sydney: University of Sydney, 1993. PhD thesis.

14 Fortney SM, Vroman NB. Exercise, performance and temperature control: temperature regulation during exercise and implications for sports performance and training. Sports Med 1985;2:8-20.

15 Clapp JF. The changing thermal response to endurance exercise during pregnancy. Am $¥$ Obstet Gynecol 1991; 165:1684-9.

16 McMurray RG, Katz VL, Meyer-Goodwin WE, Cefalo RC. Thermoregulation of pregnant women during aerobic exercise on land and in the water. $A m \mathcal{F}$ Perinatol 1993;10:178-82.

17 O'Neill ME, Cooper KA, Hunyor SN, Boyce ES. "Pseudo" fetal bradycardia during maternal exercise. $\mathcal{F}$ Appl Physiol 1987;62:849-50.

18 Carpenter MW, Sady SP, Hoegsberg B, Sady MA, Haydon $\mathrm{B}$, Cullinane EM, et al. Fetal heart rate response to maternal exertion. $\mathcal{F} A M A$ 1988;259:3006-9.

19 Resch BA, Papp JG. The effect of changes in temperature on spontaneous heart rate of isolated surviving human on spontaneous heart rate of isolated surviving

20 Walker D, Walker A, Wood C. Temperature of the human fetus. F Obstet Gynaecol Br Cwealth 1969;76:503-11.

21 Hauth JC, Gilstrap LC, Widmer K. Fetal heart rate reactivity before and after maternal jogging during the reactivity before and after maternal jogging during
third trimester. Am $\mathcal{f}$ Obstet Gynecol 1982;142:545-7.

22 Lotgering FK, Gilbert RD, Longo LD. Exercise responses in pregnant sheep: oxygen consumption, uterine blood flow and blood volume. $\exists$ Appl Physiol 1983;55:834-41.

23 Webb KA, Wolfe LA, Tranmer JE, McGrath MJ. Pregnancy outcome following physical fitness training [abstr]. Can $\mathcal{F}$ Sport Sci 1988;13:93-4P. 